\title{
Espace, temps, objet et causalité : thèmes et variations
}

\section{Louis Allix}

\section{(2) OpenEdition}

1 Journals

\section{Édition électronique}

URL : http://journals.openedition.org/philosophiascientiae/677

DOI : 10.4000/philosophiascientiae.677

ISSN : $1775-4283$

\section{Éditeur}

Éditions Kimé

\section{Édition imprimée}

Date de publication : 1 octobre 2011

Pagination : $35-46$

ISBN : 978-2-84174-569-2

ISSN : 1281-2463

\section{Référence électronique}

Louis Allix, «Espace, temps, objet et causalité : thèmes et variations », Philosophia Scientiæ [En ligne], 15-3 | 2011, mis en ligne le 01 octobre 2014, consulté le 02 novembre 2020. URL : http:// journals.openedition.org/philosophiascientiae/677 ; DOI : https://doi.org/10.4000/ philosophiascientiae.677 


\title{
Espace, temps, objet et causalité : thèmes et variations
}

\author{
Louis Allix \\ Université de Reims-Champagne-Ardenne (France)
}

Résumé : Les principes fondamentaux régissant les rapports entre l'espace, le temps, l'objet et la causalité sont présentés et examinés. Il est découvert, par des expériences de pensée successives, que l'abandon de l'un ou l'autre de ces principes permettrait peut-être de résoudre de façon neuve des difficultés classiques de la philosophie comme la flèche de Zénon, Achille et la tortue ou le bateau de Thésée. Sont révélés à cette occasion plusieurs asymétries importantes existant entre l'espace et le temps, dans leurs rôles respectifs visà-vis des objets matériels et de la relation causale. Le rapport entre le temps et la causalité est, enfin, plus particulièrement étudié.

Abstract: The core principles ruling the relations between space, time, material objects and causality are introduced and examined. Through successive thought experiments, each of these principles is abandoned, revealing new solutions to traditional philosophical problems such as Zeno's Arrow, Achilles' Paradox and the Ship of Theseus. Several differences between space and time in the way they connect to material objects and causality are uncovered. More specifically, the reduction of time to causality is analyzed and criticized.

Quatre principes fondamentaux décrivent, de prime abord, les liens existant entre le temps, l'espace et l'objet matériel.

1. À chaque moment de son existence, un objet occupe un emplacement dans l'espace;

2. En chacun de ses emplacements, l'objet demeure un ou plusieurs instants ;

3. Un même objet ne peut pas être au même moment en deux emplacements spatiaux différents ;

4. Deux objets différents ne peuvent pas être au même moment sur le même emplacement spatial.

i. Le premier principe assure qu'un objet est dans l'espace de façon continue et non pas intermittente. Il n'est pas possible qu'il apparaisse en un lieu puis disparaisse - en n'étant, donc, plus nulle part 
- puis de nouveau apparaisse, soit dans le premier lieu, soit dans un autre. Ce principe vaut pour toutes les entités qui sont dans l'espace. Ainsi, par exemple, les partisans des champs ou ceux pour qui l'objet n'est qu'une collection de parties temporelles (temporal parts) adoptent des principes similaires : un champ ne peut pas avoir une existence intermittente dans l'espace, ni une collection de parties temporelles former une suite temporellement discontinue. De fait, dès qu'une chose, quelle qu'elle soit (objet, événement, champ, universel, cause, etc.) est dans l'espace, elle l'est de façon continue.

ii. Le deuxième principe fait à la fois (a) qu'un objet ne peut pas être dans l'espace hors du temps et (b) qu'il peut avoir une existence, non pas seulement instantanée, mais prolongée au même endroit (ce qui le différencie, par exemple, d'une partie temporelle d'objet).

iii. Le troisième principe fait qu'il faut une pluralité de moments pour que se multiplient les lieux où se trouve un objet : ce dernier doit en effet se déplacer pour occuper une multiplicité d'emplacements et cela exige du temps. De même, le troisième principe assure que, si les objets se multiplient, le nombre des emplacements se multiplie au même rythme et non pas, par exemple, plus vite.

iv. Le quatrième principe fait qu'à chaque endroit de l'espace, il y a au maximum un objet, lequel occupe, donc, son lieu de façon exclusive, comme s'il résistait à la pénétration de son emplacement par une autre chose.

Les deux premiers principes ci-dessus sont communs à la fois aux objets et aux universaux aristotéliciens qui sont, eux aussi, situés dans l'espace de façon continue et persistante. En revanche, les troisième et quatrième principes (dont on remarquera, au passage, la symétrie de structure) font la différence entre ces entités, puisque les universaux, à la différence des objets, ne les respectent pas, pouvant être simultanément en plusieurs lieux différents ou bien occuper à plusieurs le même emplacement spatial. Ainsi, les objets matériels sont individualisés par leur emplacement dans l'espace, mais non pas les universaux.

Des raisons de sens commun, fondées sur l'observation des objets macroscopiques, nous conduisent à considérer ces quatre principes comme à la fois évidents et non révisables. Cependant, nous savons bien que la science tourne souvent le dos au sens commun et ne se fonde pas sur le comportement des objets au quotidien pour statuer sur le réel. Nous ne pouvons donc pas accepter sans discussion ces principes. On répondra que les sciences, elles aussi, rejettent aujourd'hui la possibilité que l'objet puisse occuper deux lieux différents au même moment ou bien qu'il puisse n'avoir qu'une présence intermittente dans l'espace. On répondra cependant à cela que ce que disent les scientifiques sur la réalité n'a pas un statut définitif, étant par nature révisable, et que, par ailleurs, les hypothèses en apparence les plus incongrues ont 
parfois trouvé dans le passé des applications inattendues dans les sciences. Le réalisme scientifique ne nous oblige donc pas à considérer que les entités qui sont, actuellement, considérées comme étant les éléments ultimes de la réalité par les micro-physiciens, ont, dans leur être comme dans leurs caractéristiques essentielles, un statut définitif.

\section{Quelques expériences de pensée}

Demandons-nous, par conséquent, si nous ne pourrions pas abandonner l'une ou l'autre de ces quatre propositions, en commençant par la première d'entre elles, qui interdit qu'un objet puisse être seulement de façon intermittente dans l'espace. Imaginons ainsi qu'une entité matérielle puisse « quitter» momentanément l'espace pour, ensuite, y revenir. Cette supposition est, à première vue, tout à fait extravagante et peut se voir opposer immédiatement bien des objections mais considérons-nous comme étant, non pas dans un contexte de justification, mais dans un contexte de découverte et examinons en quoi cette proposition pourrait avoir un côté positif. Or, l'on perçoit immédiatement que cette hypothèse audacieuse nous donne une solution nouvelle au paradoxe de la flèche de Zénon, cette flèche tirée par un arc qui change tout le temps d'emplacement, mais est pourtant, à chaque instant, immobile [Aristote Physique, VI, 9]. En effet, si la flèche de Zénon pouvait n'être dans l'espace que par intermittence, et si c'était son « déplacement » qui se faisait hors de l'espace, nous pourrions comprendre pourquoi son mouvement n'est jamais saisi comme tel, à quelque moment que cela soit : il y aurait, en effet, un moment où le trait ne serait plus dans l'espace puis un moment où il réapparaîtrait en un autre endroit, sans qu'il y ait eu, à proprement parler, de passage d'un lieu à un autre, sans translation donc, mais seulement, littéralement, un dé-placement puis un re-placement dans l'espace.

Certes, nous ne pourrions pas percevoir - et peut-être même concevoir clairement - ces quantas de déplacement mais cela n'est pas gênant, puisque nous savons qu'imperceptibilité, et même inconcevabilité, ne signifient pas irréalité. Bien sûr, rien aujourd'hui ne nous assure que cette solution, qui met à mal une bonne partie de ce que nous croyons être l'espace et le temps dans leur rapport avec l'objet matériel, sera prise au sérieux dans un avenir plus ou moins lointain par les scientifiques, mais rien non plus ne nous assure aujourd'hui que ce ne sera jamais le cas. De même, rien ne prouve que la philosophie n'aura pas besoin un jour, indépendamment de l'évolution des sciences, d'envisager une telle hypothèse. La résolution d'un problème philosophique a parfois un coût élevé.

En continuant notre expérience de pensée, imaginons maintenant que nous abandonnions non plus le premier principe exposé plus haut mais le deuxième et posons, donc, qu'un objet peut être dans l'espace sans être dans le temps. 
On remarquera tout de suite que, là encore, le problème de la flèche de Zénon pourrait trouver une solution originale : cet objet occuperait maintenant une partie de ses emplacements sans être dans le temps et pourrait donc être, à la fois, à chaque instant immobile et en mouvement, puisque ce mouvement de déplacement aurait lieu, précisément, en dehors du temps. Cette hypothèse est, il est vrai, encore plus étrange que la précédente et, même, semble exiger une modification si radicale de notre concept de temps qu'elle apparaît à première vue comme n'étant pas du tout une solution au problème de la flèche de Zénon. Nous sommes cependant peut-être ici, comme plus haut, devant une règle de notre « grammaire existentielle », qui pourrait tout de même être changée, si nous modifions en profondeur l'architecture de nos réseaux de concepts.

L'abandon des deux premiers principes présentés supra nous offre donc de nouvelles pistes pour résoudre l'énigme de Zénon. Examinons maintenant si, de la même manière, la suppression du troisième principe (qui pose qu'un même objet occupe, à chaque moment, au maximum un emplacement) pourrait fournir encore une solution à notre problème. Or, c'est effectivement le cas. Si, en effet, la flèche de Zénon était à chaque moment de son existence en au moins deux emplacements à la fois, elle pourrait être tout d'abord en un lieu puis à l'instant suivant en un autre lieu, tout en étant à chaque fois immobile et cependant toujours, aussi, en mouvement. Dans un premier moment, elle occuperait, par exemple, les deux emplacements non-contigus $E_{1}$ et $E_{2}$ ensemble puis, l'instant suivant, serait sur les deux emplacements distincts $E_{2}$ et $E_{3}$. La translation de la flèche consisterait, ainsi, en une succession discrète de couples d'emplacements, ayant un élément en commun avec le couple suivant, ce qui permettrait à la flèche d'être à la fois immobile à chaque instant et pourtant, toujours en « déplacement », puisqu'elle serait en permanence en deux lieux distincts, simultanément. Cette hypothèse est bien sûr, comme les deux précédentes, très contestable. Mais, elle n'est pas contradictoire. En outre, elle n'est pas à première vue rejetable de façon expérimentale puisqu'il faudrait, pour faire cela, que nos instruments de mesure soient un jour capables de discerner exactement les limites des emplacements spatiaux de la réalité, ce qui n'est nullement certain.

Si l'on pousse maintenant l'hypothèse un peu plus loin, en rejetant encore une fois le troisième principe, et posons qu'un objet occupe à chaque instant, non plus deux, mais un nombre infini d'emplacements spatiaux contigus et partiellement superposés, c'est cette fois-ci le paradoxe d'Achille et la tortue qui pourrait peut-être trouver une solution originale. La question ne se poserait plus, en effet, de savoir comment Achille peut commencer d'avancer pour rejoindre la tortue, alors qu'il n'y a pas de points voisins dans l'infinité des points géométriques qui le séparent de cette dernière, puisqu'Achille serait dès le premier instant sur une quantité infinie d'emplacements, avant même de commencer d'avancer! 
La suppression du troisième principe a encore d'autres avantages. Elle permet notamment de dissoudre le paradoxe du bateau de Thésée, cette embarcation qui semble être à la fois en cours de rénovation dans le port du Pirée et en voie de reconstitution, avec les parties détachées du premier navire, sur une cale sèche [Hobbes 1656, 136-137]. Le même bateau ne peut pas en effet avoir été, à la fois, restauré à son emplacement dans le port du Pirée et reconstruit dans un bassin de radoub, si un objet ne peut pas être au même moment en deux emplacements différents. Mais, si le troisième principe est abandonné et qu'il devient possible pour un même objet d'être simultanément en deux lieux distincts, il n'est plus nécessaire de décider, entre les deux navires, lequel est le bateau de Thésée. Il n'y a plus qu'une seule embarcation, constituée de deux parties distinctes, situées en deux emplacements différents dans l'espace et la difficulté initiale disparaît.

On dira - et l'on aura raison de le faire - qu'il existe des solutions beaucoup plus élégantes et beaucoup plus plausibles au paradoxe du bateau de Thésée (comme du reste au problème de la flèche de Zénon vu plus haut) [Lowe 1983], [Sainsbury 1988, 23]. Mais, ces solutions qui nous satisfont aujourd'hui seront peut-être un jour rejetées. En outre, on peut imaginer d'autres situations où l'abandon du troisième principe pourrait être utile pour supprimer des difficultés liées à la question de l'identité des objets.

Imaginons ainsi, par exemple, que nous découvrions un jour, dans des conditions expérimentales particulières (à la suite, par exemple, de fissions atomiques déclenchées par des dispositifs expérimentaux sophistiqués), que des couples de corpuscules élémentaires, situés en des emplacements spatiaux très éloignés l'un de l'autre, naissent en même temps et aient toujours, exactement, à la fois les mêmes propriétés actuelles, dispositionnelles et, même, modales (comme, par exemple, des conditions identiques d'apparition et de disparition). Imaginons, de même, qu'à chaque fois que l'un des deux éléments de ces couples de particules, à la suite d'une cause extérieure, connaîtrait une modification particulière, la particule qui lui est associée subirait instantanément un changement identique. On voit tout de suite que si l'on ne souhaite pas faire intervenir de la causalité instantanée pour expliquer ces concomitances systématiques à grande distance, il pourrait être intéressant de poser l'hypothèse - ne serait-ce que pour pouvoir mieux la rejeter ensuite - que les deux objets en réalité n'en font qu'un. Ainsi, un changement dans une des deux particules ne serait pas la cause du changement dans l'autre, mais ces deux changements seraient des modifications simultanées, sous l'effet d'une cause extérieure, de deux parties spatialement non-contiguës du même objet.

Cette solution semble à première vue inacceptable. Mais, le concept de causalité instantanée pose peut-être aujourd'hui — ou posera en tout cas un jour — suffisamment de difficultés pour qu'il faille déjà envisager de lui préférer l'hypothèse que, dans certaines circonstances, une même entité matérielle peut être au même moment à deux emplacements différents de l'espace. Cela ferait 
de certains objets des sortes de champs discontinus ayant une existence transspatiale, à la manière dont, par leur persistance dans le temps, ils possèdent une existence trans-temporelle (i. e. s'étalant dans le temps plutôt que sur un seul instant). Ces «monstres », aujourd'hui inacceptables, seront peut-être un jour intégrés dans nos théories philosophiques ou dans nos explications scientifiques les plus informées ${ }^{1}$.

Nous avons donc vu que l'abandon des trois premiers principes régissant les rapports entre l'espace, le temps et l'objet pourrait fournir des solutions nouvelles à des problèmes classiques de philosophie. Si nous examinons maintenant le quatrième principe (qui pose qu'il ne peut pas y avoir, au même moment, deux objets différents sur le même emplacement spatial), nous pouvons aussi constater que son abandon, là encore, nous procurerait une solution au problème du bateau de Thésée. En effet, désormais, il ne serait plus nécessaire de réduire les deux bateaux à un seul, comme plus haut, ou d'en choisir un des deux comme étant le bateau authentique : ces deux embarcations au départ coexisteraient au même emplacement dans le port du Pirée, avant le processus de réparation de l'une et de reconstruction de l'autre, puis se sépareraient et occuperaient deux emplacements différents, le quai du port pour le bateau rénové et une cale sèche pour le deuxième bateau.

Certes, de nouveau la solution est extrême puisqu'elle entraîne qu'à chaque fois que nous sommes devant quelque chose, nous ne pouvons savoir s'il y a là un ou plusieurs objets que si nous connaissons l'historique complet de ce qui est présent devant nous, connaissance qu'il est bien sûr impossible d'avoir sur le moment. Cette solution sera donc rejetée par toute personne de bon sens, mais, encore une fois, elle n'est pas logiquement absurde et rien ne nous oblige à penser qu'elle sera toujours totalement inenvisageable.

En outre, elle permet de résoudre - et peut-être, même, de façon plus plausible - cette autre difficulté classique liée à l'identité des objets et qui consiste à comprendre comment un bloc de bronze et la statue qui est faite à partir de celui-ci - qui ne sont pas le même objet, puisqu'ils ont des conditions d'existence différentes, ne venant pas au monde au même moment et disparaissant dans des circonstances différentes - peuvent cependant partager le même emplacement. La solution la plus courante à ce problème consiste à faire du bloc de bronze quelque chose qui n'a pas le statut d'objet mais appartient à un genre d'être inférieur, ce qui fait qu'il peut partager son emplacement avec l'objet en bonne et due forme qu'est la statue [Wiggins 1980, 30 sq.]. Mais, on peut préférer à cette explication la réponse qui consiste à faire des deux entités des objets à part entière, sans hiérarchie entre eux, et à accepter, contre le sens commun, qu'à partir du moment où la statue

1. Cf. aussi, plus loin, la note $\mathrm{n}^{\circ} 5$. 
vient à l'existence dans le bloc de bronze, ces deux objets matériels occupent le même emplacement.

\section{Des rôles différents pour l'espace et pour le temps}

Nous avons donc vu un certain nombre d'avantages qu'il pourrait y avoir à changer radicalement notre appréhension des rapports existant entre les objets matériels, l'espace et le temps. Encore une fois, de très nombreuses objections peuvent être faites à ces propositions audacieuses, ne serait-ce que parce qu'elles s'opposent si formidablement à nos intuitions les plus profondes concernant ces trois catégories fondamentales de la réalité. Mais, il peut être intéressant, plutôt que de soulever ces objections, de prolonger encore un peu notre Gedankenexperiment.

Remarquons d'abord que les quatre principes présentés au début de cet article montrent que l'espace et le temps jouent des rôles différents par rapport à l'objet. L'espace donne à l'objet son individualité en lui fournissant, à tout instant, un emplacement unique, qu'il occupe exclusivement, mais c'est le temps qui donne à l'objet son indépendance par rapport à l'espace, puisque c'est grâce au déploiement du temps en une multiplicité indéfinie de moments que l'objet peut se déplacer et donc, à la fois, occuper plusieurs emplacements différents et, par ce déplacement, être sur un emplacement précédemment occupé par une autre chose. À la fois, donc, (a) la présence au même emplacement de deux objets et (b) la présence d'un même objet en deux emplacements différents sont rendus possibles par l'existence du temps

A contrario, une pluralité d'emplacements spatiaux, si elle est nécessaire pour que deux objets existent dans le même moment de temps, ne l'est pas pour qu'un objet existe en deux moments différents. Un objet peut exister sur plus d'un instant tout en restant au même endroit : c'est, de fait, ce qu'énonce le deuxième principe vu plus haut. Et, plus généralement, il n'est pas impossible, pour la même chose, d'être plus d'une fois au même emplacement, soit par retour sur l'un de ses emplacements antérieurs, soit par son maintien plus d'un instant sur le même emplacement spatial (ce que, au vrai, nous pouvons constater immédiatement dans le monde macroscopique : un objet peut revenir à l'emplacement spatial qu'il occupait précédemment, ou bien demeurer une quantité de temps indéfinie, au même endroit).

Cependant, il peut être, là encore, intéressant de s'opposer, comme plus haut, au consensus et de poser qu'un objet ne peut pas être au même emplacement sur plus d'un moment, à la manière par exemple dont une entité, dans le 
continuum espace-temps quadridimensionnel, ne peut jamais être deux fois au même emplacement spatiotemporel (défini par ses quatre coordonnées). Ainsi, un objet dans l'espace tridimensionnel ne pourrait plus changer d'emplacement sans changer d'identité, ce qui ferait de lui une sorte de temporal part, mais à durée d'existence toujours minimale. De la sorte, un nouveau principe serait introduit : « un même objet ne peut pas être, en deux moments différents, sur le même emplacement spatial », qui serait très proche structurellement du troisième principe : « un objet ne peut pas être, au même moment, sur deux emplacements spatiaux différents ».

\section{Le changement et la causalité}

L'espace et le temps ont donc des rapports différents à l'objet. Mais, le temps et l'espace jouent aussi des rôles très différents vis-à-vis du changement. Le temps est, en effet, nécessaire pour qu'il y ait du changement, puisque ce dernier n'est possible que s'il existe des relations temporelles d'antériorité/postériorité (un «avant » et un « après », dit Aristote [Physique, IV, 14]), alors que l'espace, lui, n'est pas nécessaire pour qu'il y ait du changement, puisqu'on peut concevoir du changement hors de l'espace, par exemple dans un univers mental. Le concept de changement requiert, de fait, seulement la présence, à un moment donné, d'une propriété, puis, au moment suivant, d'une autre propriété, sans que référence explicite ne soit faite à l'espace ou à des emplacements particuliers dans l'espace.

Le temps et l'espace n'ont donc pas le même rapport au changement, ce qui fait, aussi, que leur rapport à la causalité est différent. Le temps étant, en effet, une condition nécessaire pour qu'il y ait du changement, il est par conséquent nécessaire aussi pour qu'il y ait de la causalité, puisque cette dernière ne peut exister sans ses relata, c'est-à-dire sans les changements de propriétés qu'elle relie. En revanche, l'espace n'est pas nécessaire pour qu'il y ait de la causalité puisque, là encore, on peut aisément concevoir qu'il puisse y avoir de la causation hors de l'espace comme, par exemple, dans un milieu mental ${ }^{2}$.

La causalité, toutefois, est dans un rapport analogique spécial avec l'espace parce qu'elle joue, vis-à-vis du couple cause-effet qu'elle connecte, un rôle similaire à celui que joue l'objet par rapport à l'espace. Ainsi, au principe qu'un même objet ne peut pas être en plus d'un emplacement au même moment, correspond le principe qu'une relation causale ne peut pas lier plus de deux événements à la fois. De même, à la proposition qu'il ne peut pas y avoir, au même moment, plus d'un objet sur un seul emplacement répond la proposition qu'il ne peut pas y avoir plus d'une relation causale entre deux événements.

2. Le fait que l'on puisse concevoir qu'il existe de la causation mentale n'entraîne pas, bien sûr, que l'on croie que cela existe. 
La causalité est, de la sorte, par rapport au couple d'événements qu'elle relie, comme est l'objet par rapport à chacun des lieux qu'il occupe dans l'espace.

Cela a pour conséquence que, si nous pouvons examiner de façon critique les principes qui régissent l'objet dans son rapport avec l'espace, nous pouvons peut-être faire de même, à propos des principes gouvernant le rapport existant entre la causalité et les deux événements qu'elle unit. Et, de la même manière que nous avons pu remettre en question les quatre principes présentés au début de cet article à propos de l'objet matériel, nous pouvons faire un geste théorique similaire à propos de la causalité et envisager, par exemple, qu'en un même instant il puisse y avoir plus de deux événements reliés par une relation causale, ou bien plus d'une relation causale entre deux événements. Ainsi, une cause suffisante - et non pas seulement partielle - pourrait avoir, au même instant, des effets en une multiplicité d'emplacements spatiaux différents. De même, un effet pourrait avoir différentes causes, toutes suffisantes, en plusieurs endroits différents de l'espace.

Cette complexité des relations causales entre événements, certes, violerait le principe de simplicité (simplex sigillum veri). En outre, ces cas de surdétermination causale systématique - où deux événements pourraient être liés par plusieurs relations causales, chacune suffisante - contrediraient le principe d'Ockham. Mais, nous aurons peut-être un jour besoin, pour nos besoins théoriques, d'admettre qu'il puisse y avoir précisément des cas de surdétermination causale systématique ou bien, encore, des causes ayant simultanément une pluralité d'effets en différents emplacements de l'espace.

\section{Causalité et temps}

La causalité entretient donc des relations différentes avec le temps et avec l'espace. En outre, parce que, d'un côté, (a) le sens de la relation causale semble être toujours le même que celui du temps et que, de l'autre, (b) la causalité et le temps sont, à première vue, tous deux conditions nécessaires pour qu'il y ait du changement, on peut être tenté de réduire ces deux catégories fondamentales à une seule d'entre elles [Tooley 1987, 225 sq.]. De surcroît, si on accepte, d'une part, (a) le principe du déterminisme, c'est-à-dire qu'une cause est suffisante pour produire son effet et, d'autre part, (b) que dès qu'il y a du temps, il y a du changement, alors le temps et la causalité deviennent tous deux, non plus seulement des conditions nécessaires, mais aussi des conditions suffisantes pour qu'il y ait du changement, ce qui ne peut que nous inciter, plus encore, à les réduire l'une à l'autre, étant difficile d'imaginer qu'un même phénomène puisse avoir deux conditions à la fois nécessaires et suffisantes pour qu'il advienne (conditions, de surcroît, indépendantes l'une de l'autre). Et, comme il est difficile, par ailleurs, de poser que le temps et la causalité peuvent exister l'un sans l'autre - il faudrait en effet, alors, pouvoir envisager 
qu'il y ait de la causalité hors du temps ou bien du temps qui agirait sur la réalité de façon non causale - la tentation de les réduire l'un à l'autre devient encore plus grande.

Cependant, on ne peut réduire le temps et la causalité à n'être la même chose que si, à la fois, (a) ils sont donc bien, tous deux, des conditions suffisantes pour qu'il y ait du changement mais aussi — et surtout - si (b) la causalité s'exerce toujours dans la même direction que le temps et jamais à contre-sens de celui-ci. La question du déterminisme débordant très largement du cadre de cet article, nous remettrons son examen à une autre occasion. Mais, nous avons des raisons de penser que, dès qu'il y a du temps, il y a du changement, ne serait-ce que parce que, s'il était possible que tout changement disparaisse pendant un certain temps, il n'y aurait aucune raison qu'ensuite des changements adviennent de nouveau, à un moment plutôt qu'à un autre. Il y aurait là quelque chose d'incompréhensiblement arbitraire. En outre, il faudrait admettre, soit qu'il y ait des causes qui n'agissent que longtemps après leur advenue — c'est-à-dire des événements qui n'auraient d'efficace causale que longtemps après leur disparition ${ }^{3}$ —, soit — pour éviter qu'il y ait de la causalité agissant comme par un saut dans le futur - que, sur une certaine durée, des absences de changement soient causes d'autres absences de changements puis, incompréhensiblement, qu'une absence de changement particulière fasse advenir causalement un changement. Tout cela est difficile à admettre. Il est donc peu plausible que du temps puisse se dérouler dans l'univers sans qu'il n'y ait, nulle part, de changement de quoi que ce soit. Ce qui nous donne de bonnes raisons de penser que le temps est condition suffisante pour qu'il y ait du changement et que, donc, temps et causalité sont la même chose ${ }^{4}$.

Mais, comme nous l'avons vu plus haut, pour pouvoir poser que temps et causalité sont la même chose, encore faut-il qu'il ne puisse pas y avoir de causalité à rebours du temps. Or, cela ne va pas de soi, malgré les apparences. Certes, il existe une grosse objection contre la backward causation : s'il y avait de la causalité du futur vers le présent ou du présent vers le passé, il faudrait que la cause agissant à contre-sens du temps ne puisse jamais produire dans le passé une suite d'effets qui rendrait impossible sa propre existence. En illustrant cela avec l'exemple d'une personne qui serait envoyée vers son propre passé [Horwich 1987, 116], cela veut dire qu'il faudrait qu'aucun de ses actes - et aucune des conséquences ultimes de ceux-ci — ne puisse rendre impossible sa propre naissance ainsi que l'en-

3. Ainsi, sans doute, qu'une ou plusieurs lois qui feraient que ces causes agissent à distance dans le temps plutôt qu'immédiatement.

4. Remarquons que, s'il est tentant de considérer temps et causalité comme étant identiques, rares sont les métaphysiciens qui cherchent à remplacer l'espace par la causalité, ne serait-ce que parce qu'il est beaucoup plus facile d'imaginer qu'il puisse exister des endroits dans l'espace où jamais rien ne change et où, donc, ne s'exerce aucune causalité, que de penser qu'il existe des moments de temps où, dans tout l'Univers, aucun changement n'a lieu et où, par conséquent, il n'y a pas de causalité. 
semble des autres événements - en quantité sans doute considérable — qui ont rendu possible son expérience de retour vers le passé. C'est extrêmement peu vraisemblable. Plus généralement, il faudrait que l'ensemble des effets produits rétroactivement dans le passé par une cause future, quelle qu'elle soit, ne puisse jamais briser les liaisons causales qui sont indispensables pour construire la suite d'événements grâce auxquels cette même action à rebours du temps a pu venir à l'existence. Il est très difficile, là encore, de concevoir cela. Il est donc très peu plausible que de la causalité à rebours du temps puisse se produire.

Mais, il faut remarquer que l'invraisemblance, même extrême, n'est pas synonyme d'impossibilité logique. Et, de fait, il n'est pas exclu qu'un jour, dans des circonstances particulières (par exemple, lors d'observations dans un laboratoire de microphysique, sur un temps très court et dans un contexte très étroit), il devienne raisonnable de poser que de la causalité du futur vers le passé a pu avoir eu lieu - parce que nous aurions, par exemple, de bonnes raisons de penser que les chaînes causales créées dans le passé par la cause rétroactive n'ont pas détruit les liaisons causales qui ont joué un rôle nécessaire dans l'advenue de cette même cause. Il reste donc logiquement possible que de la causalité puisse s'exercer dans la direction contraire à celle du temps. Par conséquent, temps et causalité ne peuvent pas être identiques ${ }^{5}$.

On peut en outre avancer encore un argument contre l'idée que le temps et la causalité sont assimilables l'un à l'autre. En effet, parce qu'un changement exige un «avant» et un «après », il faut au moins deux moments de temps pour qu'il existe. Et, comme la causalité exige à son tour deux changements pour avoir lieu (le premier changement étant la cause et le deuxième, l'effet), il faut au moins quatre moments de temps pour qu'il y ait de la causalité. Cela signifie qu'il peut exister, au moins théoriquement, des moments où le temps existe mais où n'existe encore aucune causalité ${ }^{6}$. Temps et causalité ne peuvent donc pas, encore une fois, être la même chose.

5. Par ailleurs, même si de la causalité à rebours du temps avait lieu, il ne peut y avoir eu deux passés, l'un ayant existé sans cette intervention causale et l'autre, avec. Cela signifie que, si une personne se rend dans le passé, elle devra avoir été présente deux fois sur Terre au moment où ce retour a eu lieu : à la fois (a) comme la personne qui aurait de toutes les façons été présente s'il n'y avait pas eu de causalité depuis le futur et (b) comme la même personne qui est arrivée du futur. Nous sommes de nouveau devant un cas où le même objet est présent, au même moment, en deux endroits différents de l'espace, en violation du troisième principe présenté plus haut. Et, comme il n'y a pas d'impossibilité logique à ce que cette sorte de situation ait lieu, cela signifie qu'il n'est pas contradictoire de dire qu'un objet matériel peut être, au même moment, en deux emplacements spatiaux différents.

6. On dira, certes, que s'il existe de la causalité instantanée, la cause et l'effet ont lieu au même moment et il suffit donc de deux moments de temps pour que la causalité s'exerce. Mais, c'est encore un moment de trop pour que l'on puisse identifier temps et causalité. 
Nous ne pouvons pas, par conséquent, changer l'ensemble de nos conceptions concernant l'espace et le temps. Cependant, nos expériences de pensées successives nous ont montré que les propositions les plus générales portant sur l'espace, le temps et la causalité, dans leur rapport avec l'objet matériel, étaient peut-être modifiables. Nous avons vu, en particulier, qu'en raison des gains théoriques qu'il était possible d'obtenir, notamment pour résoudre des énigmes classiques comme la flèche de Zénon, Achille et la tortue ou le bateau de Thésée, l'abandon de l'un ou l'autre des quatre principes cardinaux qui régissent les rapports existant entre ces quatre catégories fondamentales était envisageable.

On objectera que l'objet n'est peut-être plus aujourd'hui une entité pertinente pour la métaphysique ou pour les sciences, pouvant - et même devant - être remplacé par d'autres êtres comme les champs, les cordes, les parties temporelles d'objets, les tropes, etc. Cela est peut-être vrai mais, même si c'était le cas, cela n'invalide pas les présentes réflexions. Cela nous oblige toutefois à prolonger les thought experiments présentés ici, et à explorer aussi, à propos de ces autres entités, les relations qu'elles entretiennent avec l'espace, le temps et la causalité. Ce sera l'objet d'une réflexion ultérieure.

\section{Bibliographie}

Hobbes, Thомаs

1656 The English Works of Thomas Hobbes, Vol. I, Londres : John Bohn, 1839.

Horwich, PaUl

1987 Asymmetries in Time : Problems in the Philosophy of Science, Cambridge, Mass. : MIT Press.

LOWE, E. J.

1983 On the identity of artifacts, Journal of Philosophy, 80, 222-232.

SAInsbury, Mark

1988 Paradoxes, Cambridge : Cambridge University Press.

Tooley, Michael

1987 Time, tense and Causation, Oxford : Oxford University Press.

Wiggins, DAVID

1980 Sameness and Substance, Oxford : Blackwell. 